\title{
New Anti-Neoplastics Obtained by a Molecular Connectivity Method
}

\author{
S. Villagra ${ }^{1}$, E. Jáuregui and J. Gálvez ${ }^{2}$ \\ ${ }^{1}$ Universidad Nacional de San Luis, Fac. de Qca., Bioqca. y Fcia, Cátedra de Qca. Gral. Chacabuco y \\ Pedernera. (5700) San Luis. Argentina \\ E-mail: villagr@unsl.edu.ar \\ ${ }^{2}$ Unidad de Diseño de Fármacos y Conectividad Molecular. Fac. de Fcia., Universidad de Valencia, \\ Spain
}

\begin{abstract}
Molecular Connectivity is a method, which allows to discriminate different pharmacological activities on the basics of numeric parameters of the molecules in study, related with specific and exclusive indexes. In the present study, we propose two potentially anti-neoplasic compounds: tricromil and zomepirac, by analyzing more than a hundred different chemicals.
\end{abstract}

\section{Introduction}

At the present, the most common methods for pharmacological compound design include the use of physical-chemical descriptors from QSAR methology, along with the possible complementary addition of quantum mechanics calculations or graphic methods based on molecular mechanics.

An alternative method, based on molecular topology and called «Molecular Connectivity», consists on numerically characterizing the molecule in study by a series of indexes that are specific and exclusive for each one.

The aim of the method is to obtain multi-lineal correlation between physical, chemical and biological properties of molecules, after their topology quantification. For this, correlation functions are obtained between these properties (connectivity functions) and a series of descriptors called topological indexes.

This technique has been applied to a group of diverse anti-neoplasic compounds finding connectivity functions that are capable of discriminating if a particular compound has cytotoxic activity or not.

\section{Methods and Calculations}

In this work, 62 indexes were used for determination of connectivity functions. Hence, regression 
functions that describe each property were obtained by correlation of experimental values of properties with use of statistic packages for multi-lineal correlation.

In order to classify the chemicals by their anti-neoplasic activity, an equation was defined by use of discriminating lineal analysis and working on a database of about 12 thousand compounds. A large group of chemicals were selected and distributed into two subgroups: one with contrasted antineoplasic activity and another for which this activity has not been yet described.

Using connectivity indexes, correlation functions were chosen for different properties and used as filters for selection of possible anti-neoplasics. From application of the discriminating equation of choice, two pharmacological compounds, for which no anti-neoplasic activity had been described, were chosen.

\section{Results and Discussion}

The chosen discriminating function was:

$\mathrm{D}=-9.06457-1.5237^{2} \mathrm{X}^{\mathrm{V}}+2.06966{ }^{4} \mathrm{X}_{\mathrm{p}}-18.54615 \mathrm{~J}_{2}+34.43409 \mathrm{~J}_{2}^{\mathrm{V}}$

Once applied to the selected group of compounds, it correctly classified $90 \%$ of the active and 93.1\% of the inactive compounds. The use of this method allows finding new active compounds within series defined by particular structural conditions.

In the present work, two potential cytostatic compounds were selected:<smiles>O=c1c(Br)coc2ccccc12</smiles>

zomepirac

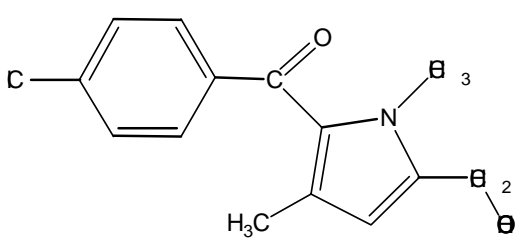

tricromil

The first of these compounds has an activity defined as anti-spasmodic and coronary vasodilator, while tricromil is known as an analgesic and anti-inflammatory.

Acknowledgements: This study has been done with funds from the UNSL and with support from Univ. De Valencia, Spain, through the program for scientific cooperation that binds both institutions.

\section{References and Notes}

1. Gálvez, J.; Gómez, M.; García, R.; Castell, J. Bioorg. Med. Chem. Lett. 1996, 19, 2301.

2. García, R.; de Julián, J. J. Chem. Inf. Comput. Sci. 1998, 38 (3), 445.

3. García, R.; Gálvez, J.; Moliner, R.; García, F. Drug Invest. 1991, 3 (5), 344.

4. Galvez, J.; García, R.; Ríos, I. Bioorg.Med.Chem.Lett. 1998, 8, 477. 